\title{
The Role of Visfatin in Pregnancy, Complications and Procreation
}

\author{
Lucia Marseglia ${ }^{1}$ Sara Manti ${ }^{1}$ Gabriella D'Angelo ${ }^{1}$ Caterina Cuppari ${ }^{1}$ Vincenzo Salpietro ${ }^{1}$ \\ Martina Filippelli ${ }^{1}$ Valeria Chirico ${ }^{1}$ Eloisa Gitto ${ }^{1}$ Carmelo Salpietro ${ }^{1}$ Teresa Arrigo ${ }^{1}$ \\ ${ }^{1}$ Department of Pediatrics, University of Messina, Messina, Italy \\ Address for correspondence Sara Manti, MD, Department of \\ Pediatrics, University of Messina, Via Consolare Valeria, 98125 \\ J Pediatr Biochem 2015;5:2-7. \\ Messina, Italy (e-mail: saramanti@hotmail.it).
}

\begin{abstract}
Keywords

- visfatin

- newborns

- pregnancy

- gestational diabetes mellitus

- preeclampsia

- breast-feeding

Adipose tissue is considered an endocrine organ secreting numerous neuroendocrine and peripheral peptides, also known as "adipokines." Although, the role of adipokines, precisely visfatin, is still controversial, it was recently discovered their involvement in different mechanisms, including metabolism, inflammation, and endocrineimmunologic system. A literature search of electronic databases was undertaken for the major studies published from 1957 to present. The databases searched were: PubMed, EMBASE, Orphanet, Midline, and Cochrane Library. This review aims to emphasize the molecular and endocrine mechanisms of visfatin and its role in fetal development. This review also reviews the role of adipocytokine in the pathogenesis of inflammatory-endocrine disorders. Further research will bring new insight into linkage between visfatin and humans, during pregnancy and perinatal period.
\end{abstract}

\section{Introduction}

Adipose tissue is considered as an endocrine organ secreting numerous neuroendocrine and peripheral peptides, called adipokines (adiponectin, leptin, resistin, ${ }^{1}$ vaspin, apelin, visfatin, tumor necrosis factor- $\alpha$ [TNF- $\alpha$ ], acylation-stimulating protein [ASP], interleukin-6 [IL-6], plasminogen activator inhibitor-1 [PAI-1], and transforming growth factor- $\beta$ [TGF$\beta]^{2,3}$ ). They act as inflammatory molecules, playing a critical role in both short- and long-time energy homeostasis, metabolic processes, and body fat regulation. ${ }^{4}$

Moreover, these peptides are considered as a new link between obesity, insulin resistance, ${ }^{5}$ cardiovascular disease, hypertension, as well as hyperlipidemia. ${ }^{6}$

This review aims to emphasize the molecular and endocrine mechanisms of visfatin and its role in fetal development.

\section{Review}

Nicotinamide phosphoribosyltransferase (Nampt) has been identified originally as pre-B-cell colony-enhancing factor (PBEF), capable of promoting the maturation of B-cell precursors together with IL-7 and stem cell factor (SCF). ${ }^{7}$ It was rediscovered as an adipocytokine, also known as "visfatin," found in visceral adipose tissue, playing a role in glucose homeostasis. ${ }^{8,9}$ Nampt has also been identified as nicotinamide adenine dinucleotide (NAD) biosynthetic enzyme. However, some of its functions are controversial and are still being discussed. Nampt exists in two different types: intracellular (iNampt) and extracellular (eNampt) forms.

\section{iNampt}

In $1957,{ }^{10}$ iNampt was identified as NAD biosynthetic enzyme. ${ }^{11}$ In mammals, tryptophan, nicotinic acid, and nicotinamide are the major precursors for NAD, but nicotinamide is mainly used to synthesize it. ${ }^{12}$

Nampt promotes the transfer of a phosphoribosyl group from 5-phosphoribosyl-1-pyrophosphate (PRPP) to nicotinamide, forming nicotinamide mononucleotide (NMN) and pyrophosphate (PPi). ${ }^{13}$ NMN is then converted to NAD by nicotinamide mononucleotide adenylyltransferase. NAD plays a critical role in cellular redox reactions. Furthermore, NAD is useful for deacetylase activity of proteins and synthesis of transcriptional regulation factor (e.g., silent information regulator [SIR]). ${ }^{14,15}$ SIR, NAD-dependent enzymes called sirtuins, mediates life span extension cell (mouse fibroblasts, human vascular smooth muscle cells, cardiac myocytes, and pancreatic B cells) caused by variety of stresses as well as
Copyright $\odot 2015$ by Georg Thieme Verlag KG, Stuttgart · New York
DOI http://dx.doi.org/ 10.1055/s-0035-1554783. ISSN $1879-5390$. 
nutritional restriction. ${ }^{16}$ In fact, pancreatic B-cell-specific Sirt2-overexpressing (BESTO) mice show strongly enhanced glucose-stimulated insulin secretion (GSIS) and enhanced glucose tolerance. These findings suggest a further Namptmediated role in metabolic balance. ${ }^{17,18}$

\section{eNampt}

The molecular mechanism of secretion (through passive and/ or active secretory pathway) ${ }^{18,19}$ and the role of eNampt are not still clear. ${ }^{20}$ Three different functions have been assigned to eNampt: insulin-mimetic hormone (adipocytokine named "visfatin"), cytokine, and aNAD biosynthetic enzyme. ${ }^{21}$

Visfatin has a molecular weight of $52 \mathrm{kDa}$. The coding region of the gene encodes for 491 amino acids. ${ }^{22}$ It is synthesized by the bone marrow, liver, lungs, skeletal muscle, brain, heart, pancreas, and peripheral blood lymphocytes. ${ }^{23}$

In particular, it is mostly expressed in human visceral fat. ${ }^{24}$ In a case-control study conducted on prepubertal obese children, it was demonstrated that obese patients have higher concentrations than normal-weight people. Elevated serum visfatin levels were also positively correlated with body fat mass; in fact visfatin concentration decreases when weight loss occurs. ${ }^{25}$

Friebe and colleagues, in an experimental study, revealed that its expression is higher in adipocytes compared with preadipocytes, ${ }^{9}$ suggesting that visfatin is a differentiated adipocytes-specific molecule and its production is influenced by serum lipid levels.

The role of visfatin in glucose metabolism is still unclear.

Several case-control studies assessed that visfatin was related to several conditions such as insulin resistance, obesity, dyslipidemia, and metabolic syndrome. ${ }^{26-29}$

In fact, Tascilar and colleagues noted that visfatin is correlated with homeostatic model assessment-insulin resistance (HOMA-IR) in children. ${ }^{27}$ However, in comparative study, Berndt and colleagues noted that plasma visfatin correlates significantly with percent body fat, body mass index (BMI), and visfatin messenger RNA (mRNA) level in visceral adipose tissue, but not with visceral fat mass or waist-to-hip ratio. ${ }^{28}$ Furthermore, no relationship was observed between serum visfatin levels and fasting plasma insulin, fasting glucose and insulin sensitivity in nondiabetic subjects. In two recent studies, plasma visfatin was higher in patients with type 2 diabetes mellitus (T2DM) than in control group. $^{29}$

Probably, visfatin promotes a glucose uptake (through 3T311 on adipocytes and L6 on myocytes and glycolysis, regardless insulin). ${ }^{30}$ In fact, visfatin binds the same insulin receptor through a distinct epitope. ${ }^{24}$

Visfatin, stimulated phosphorylation of insulin receptor substrate (IRS)-1/2 and activate phosphatidyl inositol 3kinase (PI3K), induced glucose uptake, glucose transporter (GLUT)-1 protein expression, production of profibrotic factors (including type I collagenase, TGF- $\beta 1$, PAI- 1 ), increased metalloproteinase- 9 activity in THP- 1 cells (monocyte-like cell line derived from a patient with acute monocytic leukemia), and production of TNF- $\alpha$ and IL- 8 in peripheral blood mononuclear cells. ${ }^{31}$
Friebe and colleagues assessed that Nampt influenced leucocyte count and this latter is also correlated to insulin resistance and obesity. ${ }^{32}$

In fact, eNampt, proinflammatory transcription factor nuclear factor-kB (NF-kB) in a reactive oxygen species (ROS)-dependent manner, induces the adhesion of leukocytes to endothelial cells, by activating intercellular adhesion molecule (ICAM)-1 and vascular cell adhesion molecule (VCAM)-1.

These evidences suggest underlying low-grade vascular inflammation in obesity and T2DM. ${ }^{33}$

\section{Visfatin and Inflammation}

In experimental research, Moschen and colleagues demonstrated inflammatory activities of visfatin. In fact, its serum levels were higher in patients with inflammatory disease than in healthy controls ${ }^{20,30}$ (- Table 1). Visfatin seemed to induce human leukocytes and pro- and anti-inflammatory cytokine production: IL-1b, IL-1Ra, IL-6 (in synovial ${ }^{34}$ and amniotic epithelial cells), IL-8 (in neutrophils ${ }^{35}$ ), IL-10, and TNF- $\alpha$ (in monocytes, ${ }^{31}$ macrophages, ${ }^{36}$ and neutrophils). ${ }^{20,30}$ Furthermore, visfatin increased the surface expression of costimulatory molecules CD54, CD40, and CD80 (-Fig. 1). These effects involved p38 as well as MEK1 pathways as determined by inhibition with MAPK inhibitors and activation of NF-KB. In addition, macrophages, dendritic, and epithelial cells might be a source of visfatin. These data confirmed a proinflammatory role of this adipocytokine. ${ }^{30}$

\section{Visfatin and Pregnancy}

During pregnancy, changes in maternal metabolism and redistribution of maternal adipose tissue occur in response to growing and metabolic needs of fetus and placenta. In particular, during fetal development, visfatin is expressed in exocrine and endocrine tissue. iNampt is highly released by fetal membranes, amnion, myometrium, placenta, and adipose tissue.

The amnion and deciduae at term contained higher levels of PBEF mRNA. ${ }^{37}$

However, the release and regulatory mechanisms of visfatin in the fetus and the neonate remain unclear. Probably, it may be regulated by glucose and insulin, ${ }^{38}$ and it increases with progressive B-cell deterioration, insulin resistance, and maternal weight. ${ }^{29,39}$

Serum visfatin levels fluctuate with advancing gestation. Mastorakos and colleagues recorded that median concentration of visfatin were higher in the second (24-26 weeks) and third trimesters (34-36 weeks). ${ }^{40}$

Nampt seems to confer protection from apoptosis ${ }^{41}$ and to increase infection-induced response. It also promotes the release of inflammatory cytokines, such as IL- 6 and IL-8, in amnion-like epithelial cells in sepsis patients. ${ }^{20}$ Therefore, Cekmez and colleagues, in case-control study (-Table 1), proposed that visfatin could be used as a diagnostic marker similar to C-reactive protein (CRP), procalcitonin, and IL-6 in neonatal sepsis. ${ }^{42}$ 
4 Visfatin and Newborn: Underlying Mechanisms Marseglia et al.

Table 1 Descriptive table of the most important studies conducted on Visfatin function

\begin{tabular}{|c|c|}
\hline Type of study & Authors \\
\hline \multirow[t]{10}{*}{ Case-control } & Tascilar and colleagues ${ }^{27}$ \\
\hline & Chen and colleagues ${ }^{29}$ \\
\hline & Cekmez and colleagues $^{42}$ \\
\hline & Chan and colleagues ${ }^{55}$ \\
\hline & Pagano and colleagues ${ }^{56}$ \\
\hline & Yanni and colleagues ${ }^{61}$ \\
\hline & Giapros and colleagues ${ }^{62}$ \\
\hline & Cekmez and colleagues ${ }^{63}$ \\
\hline & Kim and colleagues ${ }^{72}$ \\
\hline & Demir and colleagues ${ }^{73}$ \\
\hline \multirow[t]{3}{*}{ Experimental } & Friebe and colleagues ${ }^{9}$ \\
\hline & Moschen and colleagues ${ }^{30}$ \\
\hline & Yonezawa and colleagues ${ }^{65}$ \\
\hline Cross-sectional & Mazaki-Tovi and colleagues ${ }^{51}$ \\
\hline \multirow[t]{2}{*}{ Comparative } & Berndt and colleagues ${ }^{28}$ \\
\hline & Zhaoxia and colleagues ${ }^{57}$ \\
\hline \multirow[t]{3}{*}{ Research support } & Martos-Moreno and colleagues ${ }^{25}$ \\
\hline & Mu and colleagues ${ }^{26}$ \\
\hline & Ferreira and colleagues ${ }^{70}$ \\
\hline Prospective & Malamitsi-Puchner A and colleagues ${ }^{52}$ \\
\hline Review & Garten $\mathrm{A}$ and colleagues ${ }^{20}$ \\
\hline Abstract & Milovanov and colleagues ${ }^{71}$ \\
\hline
\end{tabular}

After birth, Nampt expression decreases in exocrine tissue but remains in endocrine cells, predominately $\beta$ cells. ${ }^{23}$

Unlike term newborns, a preterm infant frequently shows glucose homeostasis imbalance. ${ }^{43}$ After hypoglycemic phase, because of limited glycogen and fat stores, hyperglycemia occurs for a condition of insulin resistance, relative insulin deficiency, and increased ratio of the glucose transporters (Glut)-1/Glut-2 in fetal tissues. ${ }^{44}$ Several studies have confirmed that alterations in circulating adipokines can be associated with adaptations to gestation, as well as in complications of pregnancy. ${ }^{45}$ Precisely, hypoadiponectinemia has been shown to be associated with insulin resistance, ${ }^{46}$ leptin may influence the "programming" of satiety, ${ }^{47,48}$ and visfatin can increase in parallel with hyperglycemia for insulin-like effect. ${ }^{49}$

In fact, several studies demonstrated that higher maternal plasma visfatin concentration is associated with insulin resistance, gestational diabetes mellitus (GDM), patients with small-for-gestational-age (SGA), ${ }^{50,51}$ and neonates with intrauterine growth restriction (IUGR). ${ }^{52}$

\section{Visfatin and Gestational Diabetes Mellitus}

It has been reported increased serum visfatin levels during pregnancy, in T1DM or T2DM, and in obese patients. ${ }^{39}$ Insulin resistance is accompanied by increased visfatin production and/or secretion, it reflect a compensatory mechanism favoring insulin deficiency. In fact, a gradually increasing insulin resistance during 1 st and 2nd trimester of pregnancy may be compensated for by a sustained increase of visfatin, an insulinomimetic molecule., ${ }^{83}$ However, visfatin did not change in a similar way during all 3 trimesters. This condition can be attributable to an increase of visfatin production by an additional source other than adipose tissue, the placenta. Therefore, the increased serum concentration observed in gestational diabetes mellitus (GDM) may result from placental oversecretion. ${ }^{54}$ Insulin resistance is physiologic in women with normal pregnancies and it is directly correlated with gestational age. Women affected by GDM showed hyperglycemia and hyperinsulinemia. Insulin response can decrease by up to $40 \%$ in late pregnancy..$^{40}$ Other studies, however, have revealed opposite effects in gestational diabetes ${ }^{55}$ and obesity. ${ }^{56}$ Zhaoxia and colleagues evaluated visfatin levels in normal pregnancy and in women with GDM. They found that visfatin levels increased following oral glucose in normal pregnancy, and this directly correlated with glycemia, cholesterol, and insulin resistance. Otherwise, serum visfatin levels were lower in women with $\mathrm{GDM}^{57}$ (-Fig. 1).

\section{Visfatin in IUGR and SGA Newborns}

Recently, a relationship between circulating maternal visfatin and fetal growth has been proposed. In the third trimester of pregnancy, Fasshauer and colleagues reported that women with fetal growth restriction (FGR) presented higher plasma maternal visfatin than with control group. ${ }^{58}$ It has been hypothesized that visceral adipose tissue is the major source of visfatin, ${ }^{8}$ and data suggest that low-birth-weight (LBW) and IUGR newborns may have increased visceral fat stores. Therefore, Malamitsi-Puchner and colleagues prospectively considered that visfatin might be considered as biomarker for metabolic syndrome in IUGR. ${ }^{52}$ However, Harrington and colleagues did not note differences in fat distribution between newborns with IUGR and control group. ${ }^{59}$ Other authors noted that cord blood visfatin concentrations did not differ between SGA and adequate-for-gestationalage (AGA) newborns. Some studies showed that cord serum visfatin is closely associated with indices of fetal size in infants from smoking mothers, but not control group. ${ }^{60}$ Otherwise, other authors achieved opposite results finding higher visfatin levels in large-for-gestational-age (LGA) newborns. In cross-sectional study Mazaki-Tovi and colleagues investigated the linkage between visfatin and risk of development of GDM and fetal size, precisely in LGA neonate. ${ }^{45}$ In response to maternal hyperglycemia, fetus develops hyperglycemia and hyperinsulinemia due to pancreatic islet cells stimulation. In LGA neonates, hyperinsulinemia in utero leads to fetal macrosomia. LGA, SGA, and LBW newborns have an increased risk for developing metabolic syndrome in adulthood. $^{49}$

Recently, however, it has been reported that preterm newborn, whether SGA or AGA, with normal BMI, did not show insulin resistance in childhood. There were no detected 


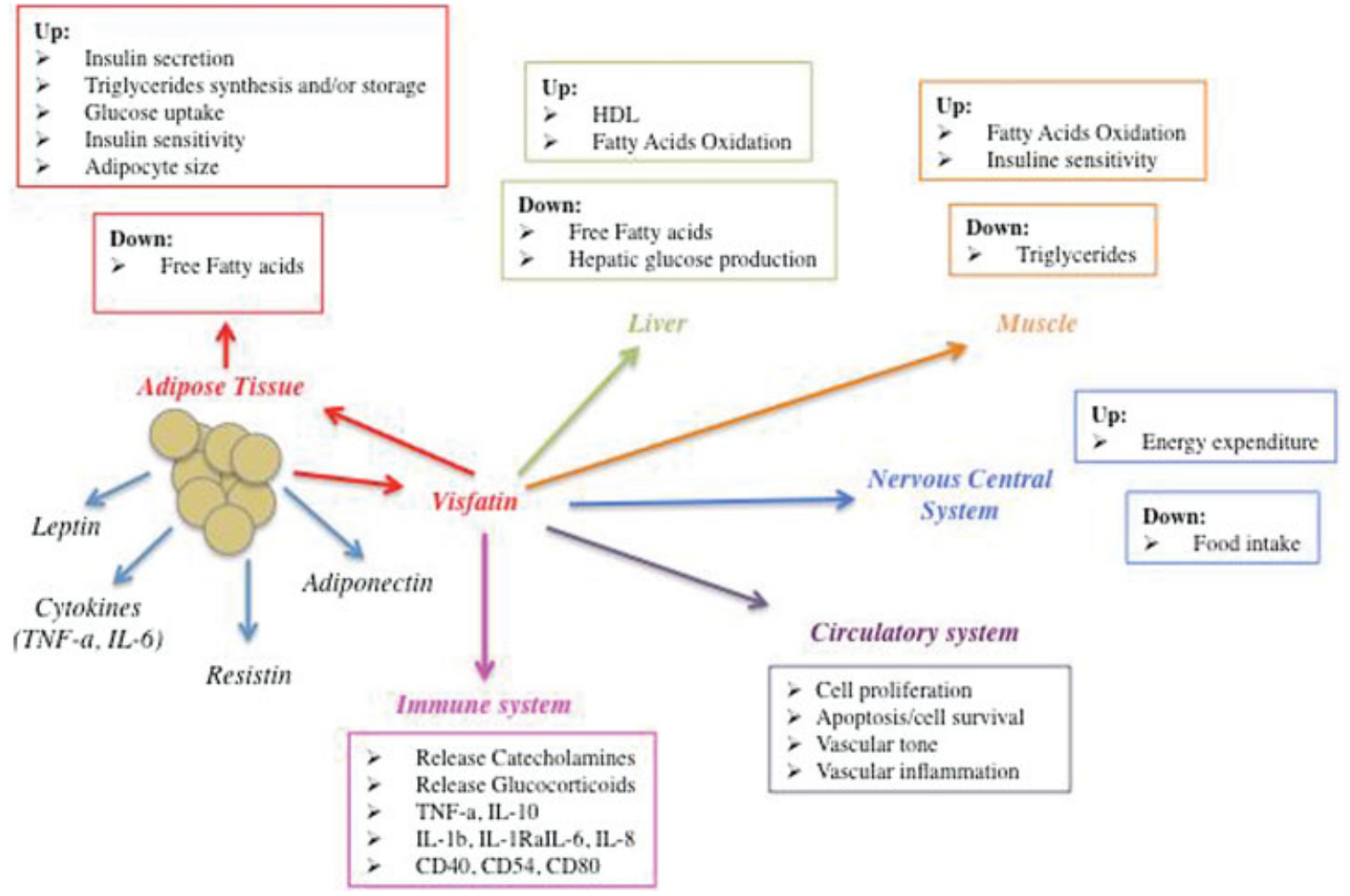

Fig. 1 Visfatin secretion and function. HDL, high-density lipoprotein; IL-6, interleukin-6; TNF- $\alpha$, tumor necrosis factor- $\alpha$.

significant differences in adipocytokine levels between the preterm SGA and AGA groups. ${ }^{61,62}$

However, in a control study, it has been suggested that, independent of being SGA or LBW, visfatin might be an early indicator of insulin resistance. ${ }^{63}$ It was also noted lower adipopectic and higher visfatin levels in LGA and SGA than AGA neonates. ${ }^{52}$ Consistently, an increased release of visfatin was demonstrated in the amnion of twins and triplets. It can be assumed maternal plasma visfatin also derived from the stretched fetal membranes of women with LGA fetuses. ${ }^{64}$ These data suggest that visfatin is a possible link between maternal and fetal environment.

\section{Visfatin and Breast-Feeding}

In the first 6 months of lactation, Yonezawa and colleagues demonstrated, in animal/experimental study, the presence of mRNA-visfatin in cloned bovine mammary gland and human breast cancer cell line. ${ }^{65}$ Moreover, milk visfatin levels are directly related to preconceptional maternal BMI. In fact, it has been found higher breast milk visfatin concentrations for a BMI greater than 28 to $30 \mathrm{~kg} / \mathrm{m}^{2}$. Visfatin seems to limit a weight loss in newborns. ${ }^{66}$

\section{Visfatin and Preeclampsia}

Preeclampsia (PE) is a multisystemic disorder of pregnancy, characterized by onset hypertension, endothelial dysfunction, and proteinuria that develop after 20 weeks of gestation in previously normotensive women. ${ }^{67}$ The precise cause of PE is still unclear, but it is believed to be likely multifactorial. The role of visfatin in PE was investigated by several studies. Circulating visfatin concentrations are increased in PE in some studies, ${ }^{68}$ whereas other investigators show similar ${ }^{52}$ or even decreased concentrations. ${ }^{69}$ Ferreira and colleagues hypothesized that visfatin develops PE, promoting an impaired placental vascularization, due to increased serum vascular endothelial growth factor (VEGF) levels ${ }^{70}$ ( - Fig. 1). Conversely, Milovanov and colleagues noted decreased expression of VEGF as well as visfatin in pregnancies complicated by PE. ${ }^{71}$ During the third trimester of pregnancy, in women affected by PE, Kim and colleagues further showed decreased placental visfatin levels. ${ }^{72}$ Although the severity of PE did not influence serum visfatin values, authors investigated relationship between PE, gestational age (SGA), and maternal circulating visfatin concentrations. These were higher in women who delivered SGA newborns than normal pregnancy and PE groups. ${ }^{73}$ However, further studies did not confirm these data. ${ }^{51}$

In conclusions, though the primary purpose of adipose tissue is energy storage, it has been also identified as an active endocrine-immune organ releasing many cytokines (adipocytokines) and hormones. In response to an autocrine and paracrine manner to impair adipocyte function, it has been reported that phenotypic changes of adipocytes occur into inflammatory response and promote a variety of diseases. Here, we have focused on visfatin expression, a recently identified adipocytokine. Although its precise function remains to be established, this review aims to emphasize the molecular and endocrine mechanisms of visfatin and to clarify its role in the pathophysiology of inflammatory-endocrine disorders, also during normal and pathologic pregnancy 
(e.g., PE, GDM, and alteration of fetal growth). Moreover, for its potential diagnostic and/or prognostic role in the prediction of any associate disease or condition, visfatin could also be considered as a new biomarker, indicating specific disorders in the neonatal metabolic profile, determining the interconnection of the different processes, and defining disease severity. In addition, several diseases could be treated by normalization and/or regulation of the proinflammatory cytokine/adipokine profile. For these novel concepts, visfatin might be promising candidates for future pharmacologic treatment strategies. Further research will bring new insight into linkage between visfatin and humans, during pregnancy and perinatal period.

\section{Authors' Contributions}

All authors read and approved the final manuscript.

\section{Conflict of Interest}

None.

\section{References}

1 Mamì C, Marseglia L, Manganaro R, et al. Serum levels of resistin and its correlation with adiponectin and insulin in healthy full term neonates. Early Hum Dev 2009;85(1):37-40

2 Rajala MW, Scherer PE. Minireview: the adipocyte-at the crossroads of energy homeostasis, inflammation, and atherosclerosis. Endocrinology 2003;144(9):3765-3773

3 Arrigo T, Chirico V, Salpietro V, et al. High-mobility group protein B1: a new biomarker of metabolic syndrome in obese children. Eur J Endocrinol 2013;168(4):631-638

4 Chirico V, Cannavò S, Lacquaniti A, et al. Prolactin in obese children: a bridge between inflammation and metabolic-endocrine dysfunction. Clin Endocrinol (Oxf) 2013;79(4):537-544

5 d'Annunzio G, Vanelli M, Pistorio A, et al; Diabetes Study Group of the Italian Society for Pediatric Endocrinology and Diabetes. Insulin resistance and secretion indexes in healthy Italian children and adolescents: a multicentre study. Acta Biomed 2009;80(1):21-28

6 Kralisch S, Bluher M, Paschke R, Stumvoll M, Fasshauer M. Adipokines and adipocyte targets in the future management of obesity and the metabolic syndrome. Mini Rev Med Chem 2007;7(1): 39-45

7 Samal B, Sun Y, Stearns G, Xie C, Suggs S, McNiece I. Cloning and characterization of the cDNA encoding a novel human pre-B-cell colony-enhancing factor. Mol Cell Biol 1994;14(2):1431-1437

8 Fukuhara A, Matsuda M, Nishizawa M, et al. Visfatin: a protein secreted by visceral fat that mimics the effects of insulin. Science 2005;307(5708):426-430

9 Friebe D, Löffler D, Schönberg M, et al. Impact of metabolic regulators on the expression of the obesity associated genes FTO and NAMPT in human preadipocytes and adipocytes. PLoS ONE 2011;6(6):e19526

10 Preiss J, Handler P. Enzymatic synthesis of nicotinamide mononucleotide. J Biol Chem 1957;225(2):759-770

11 Revollo JR, Grimm AA, Imai S. The NAD biosynthesis pathway mediated by nicotinamide phosphoribosyltransferase regulates Sir2 activity in mammalian cells. J Biol Chem 2004;279(49): 50754-50763

12 Rongvaux A, Andris F, Van Gool F, Leo O. Reconstructing eukaryotic NAD metabolism. BioEssays 2003;25(7):683-690

13 Revollo JR, Grimm AA, Imai S. The regulation of nicotinamide adenine dinucleotide biosynthesis by Nampt/PBEF/visfatin in mammals. Curr Opin Gastroenterol 2007;23(2):164-170
14 Lin SJ, Guarente L. Nicotinamide adenine dinucleotide, a metabolic regulator of transcription, longevity and disease. Curr Opin Cell Biol 2003;15(2):241-246

15 Imai S, Armstrong CM, Kaeberlein M, Guarente L. Transcriptional silencing and longevity protein Sir2 is an NAD-dependent histone deacetylase. Nature 2000;403(6771):795-800

16 van der Veer E, Nong Z, O'Neil C, Urquhart B, Freeman D, Pickering JG. Pre-B-cell colony-enhancing factor regulates NAD+-dependent protein deacetylase activity and promotes vascular smooth muscle cell maturation. Circ Res 2005;97(1):25-34

17 Moynihan KA, Grimm AA, Plueger MM, et al. Increased dosage of mammalian Sir2 in pancreatic beta cells enhances glucose-stimulated insulin secretion in mice. Cell Metab 2005;2(2):105-117

18 Revollo JR, Körner A, Mills KF, et al. Nampt/PBEF/Visfatin regulates insulin secretion in beta cells as a systemic NAD biosynthetic enzyme. Cell Metab 2007;6(5):363-375

19 Hug C, Lodish HF. Medicine. Visfatin: a new adipokine. Science 2005;307(5708):366-367

20 Garten A, Petzold S, Körner A, Imai S, Kiess W. Nampt: linking NAD biology, metabolism and cancer. Trends Endocrinol Metab 2009; 20(3):130-138

21 Sethi JK. Is PBEF/visfatin/Nampt an authentic adipokine relevant to the metabolic syndrome? Curr Hypertens Rep 2007;9(1):33-38

22 Körner A, Blüher S, Kapellen T, et al. Obesity in childhood and adolescence: a review in the interface between adipocyte physiology and clinical challenges. Hormones (Athens) 2005;4(4): 189-199

23 Kover K, Tong PY, Watkins D, et al. Expression and regulation of Nampt in human islets. PLoS ONE 2013;8(3):e58767

24 Bełtowski J. Apelin and visfatin: unique "beneficial" adipokines upregulated in obesity? Med Sci Monit 2006;12(6):RA112-RA119

25 Martos-Moreno GA, Kratzsch J, Körner A, et al. Serum visfatin and vaspin levels in prepubertal children: effect of obesity and weight loss after behavior modifications on their secretion and relationship with glucose metabolism. Int J Obes 2011;35(10):1355-1362

$26 \mathrm{Mu} \mathrm{J}$, Feng B, Ye Z, et al. Visfatin is related to lipid dysregulation, endothelial dysfunction and atherosclerosis in patients with chronic kidney disease. J Nephrol 2011;24(2):177-184

27 Taşçilar ME, Cekmez F, Meral C, et al. Evaluation of adipocytokines in obese children with insulin resistance. Turk J Pediatr 2011; 53(3):269-273

28 Berndt J, Klöting N, Kralisch S, et al. Plasma visfatin concentrations and fat depot-specific mRNA expression in humans. Diabetes 2005;54(10):2911-2916

29 Chen MP, Chung FM, Chang DM, et al. Elevated plasma level of visfatin/pre-B cell colony-enhancing factor in patients with type 2 diabetes mellitus. J Clin Endocrinol Metab 2006;91(1):295-299

30 Moschen AR, Kaser A, Enrich B, et al. Visfatin, an adipocytokine with proinflammatory and immunomodulating properties. J Immunol 2007;178(3):1748-1758

31 Dahl TB, Yndestad A, Skjelland M, et al. Increased expression of visfatin in macrophages of human unstable carotid and coronary atherosclerosis: possible role in inflammation and plaque destabilization. Circulation 2007;115(8):972-980

32 Friebe D, Neef M, Kratzsch J, et al. Leucocytes are a major source of circulating nicotinamide phosphoribosyltransferase (NAMPT)/ pre-B cell colony (PBEF)/visfatin linking obesity and inflammation in humans. Diabetologia 2011;54(5):1200-1211

33 Adya R, Tan BK, Chen J, Randeva HS. Nuclear factor-kappaB induction by visfatin in human vascular endothelial cells: its role in MMP2/9 production and activation. Diabetes Care 2008;31(4):758-760

34 Nowell MA, Richards PJ, Fielding CA, et al. Regulation of pre-B cell colony-enhancing factor by STAT-3-dependent interleukin- 6 trans-signaling: implications in the pathogenesis of rheumatoid arthritis. Arthritis Rheum 2006;54(7):2084-2095

35 Jia SH, Li Y, Parodo J, et al. Pre-B cell colony-enhancing factor inhibits neutrophil apoptosis in experimental inflammation and clinical sepsis. J Clin Invest 2004;113(9):1318-1327 
36 Iqbal J, Zaidi M. TNF regulates cellular NAD + metabolism in primary macrophages. Biochem Biophys Res Commun 2006; 342(4):1312-1318

37 Ognjanovic S, Bryant-Greenwood GD. Pre-B-cell colony-enhancing factor, a novel cytokine of human fetal membranes. Am J Obstet Gynecol 2002;187(4):1051-1058

38 Haider DG, Schaller G, Kapiotis S, Maier C, Luger A, Wolzt M. The release of the adipocytokine visfatin is regulated by glucose and insulin. Diabetologia 2006;49(8):1909-1914

39 López-Bermejo A, Chico-Julià B, Fernàndez-Balsells M, et al. Serum visfatin increases with progressive beta-cell deterioration. Diabetes 2006;55(10):2871-2875

40 Mastorakos G, Valsamakis G, Papatheodorou DC, et al. The role of adipocytokines in insulin resistance in normal pregnancy: visfatin concentrations in early pregnancy predict insulin sensitivity. Clin Chem 2007;53(8):1477-1483

41 Kendal-Wright CE, Hubbard D, Bryant-Greenwood GD. Chronic stretching of amniotic epithelial cells increases pre-B cell colonyenhancing factor (PBEF/visfatin) expression and protects them from apoptosis. Placenta 2008;29(3):255-265

42 Cekmez F, Canpolat FE, Cetinkaya M, et al. Diagnostic value of resistin and visfatin, in comparison with C-reactive protein, procalcitonin and interleukin-6 in neonatal sepsis. Eur Cytokine Netw 2011;22(2):113-117

43 van Aerde JE, Wilke MS, Feldman M, Clandinin MT. Chapter 42, Accretion of Lipid in the Fetus and Newborn. In: Polin RA, Fox WW, Abman SH, eds. Fetal and Neonatal Physiology. 4th ed. Philadelphia, PA: Elsevier Saunders; 2011:454-469

44 Blanco CL, Baillargeon JG, Morrison RL, Gong AK. Hyperglycemia in extremely low birth weight infants in a predominantly Hispanic population and related morbidities. J Perinatol 2006;26(12): 737-741

45 Mazaki-Tovi S, Romero R, Kusanovic JP, et al. Visfatin in human pregnancy: maternal gestational diabetes vis-à-vis neonatal birthweight. J Perinat Med 2009;37(3):218-231

46 Lihn AS, Pedersen SB, Richelsen B. Adiponectin: action, regulation and association to insulin sensitivity. Obes Rev 2005;6(1):1321

47 Mamì C, Manganaro R, Marseglia L, Saitta G, Gemelli M, Martino F. Plasma leptin, insulin, and neuropeptide $Y$ response to feeding in newborn infants. Arch Dis Child Fetal Neonatal Ed 2006;91(5): F389

48 Mamì C, Manganaro R, Saitta G, Marseglia L, Martino F, Gemelli M. Plasma leptin, insulin, and neuropeptide $\mathrm{Y}$ concentrations in infants. Arch Dis Child Fetal Neonatal Ed 2005;90(1):F86-F87

49 Meral C, Cekmez F, Pirgon O, et al. The relationship between serum visfatin, adiponectin, and insulin sensitivity markers in neonates after birth. J Matern Fetal Neonatal Med 2011;24(1): $166-170$

50 Krzyzanowska K, Krugluger W, Mittermayer F, et al. Increased visfatin concentrations in women with gestational diabetes mellitus. Clin Sci (Lond) 2006;110(5):605-609

51 Mazaki-Tovi S, Romero R, Kim SK, et al. Could alterations in maternal plasma visfatin concentration participate in the phenotype definition of preeclampsia and SGA? J Matern Fetal Neonatal Med 2010;23(8):857-868

52 Malamitsi-Puchner A, Briana DD, Boutsikou M, Kouskouni E, Hassiakos D, Gourgiotis D. Perinatal circulating visfatin levels in intrauterine growth restriction. Pediatrics 2007;119(6):e1314-e1318

53 Sethi JK, Vidal-Puig A. Visfatin: the missing link between intraabdominal obesity and diabetes? Trends Mol Med 2005;11(8): 344-347

54 Ferreira AF, Rezende JC, Vaikousi E, Akolekar R, Nicolaides KH. Maternal serum visfatin at 11-13 weeks of gestation in gestational diabetes mellitus. Clin Chem 2011;57(4):609-613
55 Chan TF, Chen YL, Lee $\mathrm{CH}$, et al. Decreased plasma visfatin concentrations in women with gestational diabetes mellitus. J Soc Gynecol Investig 2006;13(5):364-367

56 Pagano C, Pilon C, Olivieri M, et al. Reduced plasma visfatin/pre-B cell colony-enhancing factor in obesity is not related to insulin resistance in humans. J Clin Endocrinol Metab 2006;91(8): 3165-3170

57 Zhaoxia L, Ying W, Danqing C. Changes in visfatin levels after oral glucose tolerance test in women with gestational diabetes mellitus. Diabetes Res Clin Pract 2012;96(3):e76-e79

58 Fasshauer M, Blüher M, Stumvoll M, Tönessen P, Faber R, Stepan H. Differential regulation of visfatin and adiponectin in pregnancies with normal and abnormal placental function. Clin Endocrinol (Oxf) 2007;66(3):434-439

59 Harrington TA, Thomas EL, Frost G, Modi N, Bell JD. Distribution of adipose tissue in the newborn. Pediatr Res 2004;55(3):437-441

60 López-Bermejo A, de Zegher F, Díaz-Silva M, Vicente MP, Valls C, Ibáñez L. Cord serum visfatin at term birth: maternal smoking unmasks the relation to foetal growth. Clin Endocrinol (Oxf) 2008; 68(1):77-81

61 Yanni D, Darendeliler F, Bas F, Kucukemre Aydin B, Coban A, Ince Z. The role of leptin, soluble leptin receptor, adiponectin and visfatin in insulin sensitivity in preterm born children in prepubertal ages. Cytokine 2013;64(1):448-453

62 Giapros VI, Kiortsis DN, Evagelidou EN, et al. Visfatin levels in prepubertal children born small or large for gestational age. Horm Metab Res 2012;44(2):135-139

63 Cekmez F, Canpolat FE, Pirgon O, et al. Adiponectin and visfatin levels in extremely low birth weight infants; they are also at risk for insulin resistance. Eur Rev Med Pharmacol Sci 2013;17(4):501-506

64 Ma Y, Cheng Y, Wang J, Cheng H, Zhou S, Li X. The changes of visfatin in serum and its expression in fat and placental tissue in pregnant women with gestational diabetes. Diabetes Res Clin Pract 2010;90(1):60-65

65 Yonezawa T, Haga S, Kobayashi Y, Takahashi T, Obara Y. Visfatin is present in bovine mammary epithelial cells, lactating mammary gland and milk, and its expression is regulated by cAMP pathway. FEBS Lett 2006;580(28-29):6635-6643

66 Bienertová-Vašků J, Bienert P, Zlámal F, et al. Visfatin is secreted into the breast milk and is correlated with weight changes of the infant after the birth. Diabetes Res Clin Pract 2012;96(3):355-361

67 Sibai B, Dekker G, Kupferminc M. Pre-eclampsia. Lancet 2005; 365(9461):785-799

68 Fasshauer M, Waldeyer T, Seeger J, et al. Serum levels of the adipokine visfatin are increased in pre-eclampsia. Clin Endocrinol (Oxf) 2008;69(1):69-73

$69 \mathrm{Hu}$ W, Wang Z, Wang H, Huang H, Dong M. Serum visfatin levels in late pregnancy and pre-eclampsia. Acta Obstet Gynecol Scand 2008;87(4):413-418

70 Ferreira AF, Rezende JC, de Cassia C Oliveira R, Akolekar R, Nicolaides KH. Maternal serum visfatin at 11-13 weeks' gestation in preeclampsia. J Hum Hypertens 2013;27(4):261-264

71 Milovanov AP, Sidorova IS, Solonitsyn AN, Borovkova EI. Immunohistochemical evaluation of the distribution of vascular endothelial growth factor in the placenta, placental bed in normal pregnancy and in women with preeclampsia [in Russian]. Arkh Patol 2008;70(3):12-15

72 Kim SC, Park MJ, Joo BS, Joo JK, Suh DS, Lee KS. Decreased expressions of vascular endothelial growth factor and visfatin in the placental bed of pregnancies complicated by preeclampsia. J Obstet Gynaecol Res 2012;38(4):665-673

73 Demir BC, Atalay MA, Ozerkan K, Doster Y, Ocakoglu G, Kucukkomurcu $S$. Maternal adiponectin and visfatin concentrations in normal and complicated pregnancies. Clin Exp Obstet Gynecol 2013;40(2):261-267 